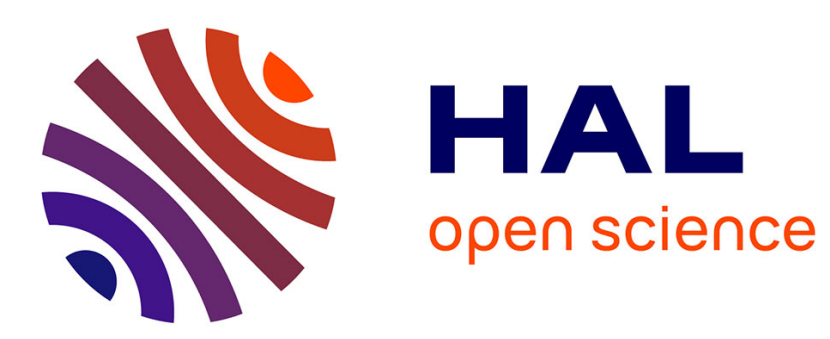

\title{
Méthodes duales pour le contact frottant
}

François Kuss, Frédéric Lebon

\section{To cite this version:}

François Kuss, Frédéric Lebon. Méthodes duales pour le contact frottant. Revue Européenne de Mécanique Numérique/European Journal of Computational Mechanics, 2007, 16 (1), pp.33-51. 10.3166/remn.16.33-51 . hal-00355088

\section{HAL Id: hal-00355088 \\ https://hal.science/hal-00355088}

Submitted on 20 Jun 2018

HAL is a multi-disciplinary open access archive for the deposit and dissemination of scientific research documents, whether they are published or not. The documents may come from teaching and research institutions in France or abroad, or from public or private research centers.
L'archive ouverte pluridisciplinaire HAL, est destinée au dépôt et à la diffusion de documents scientifiques de niveau recherche, publiés ou non, émanant des établissements d'enseignement et de recherche français ou étrangers, des laboratoires publics ou privés. 


\section{Méthodes duales pour le contact frottant}

\section{François Kuss - Frédéric Lebon}

Laboratoire de Mécanique et d'Acoustique et Université de Provence

31 Chemin Joseph Aiguier, F-13402 Marseille cedex 20

$\{$ kuss,lebon\}@lma.cnrs-mrs.fr

RÉSUMÉ. Nous présentons dans cet article, des méthodes duales pour la résolution de problèmes de contact unilatéral avec frottement de Coulomb d'un corps élastique sur un obstacle rigide. Nous abordons différentes formulations variationnelles ainsi que la discrétisation du problème. Le problème est ensuite condensé sur le bord de contact et résolu par un algorithme de relaxation de type Gauss Seidel rouge-noir. Les résultats numériques obtenus à l'aide de la méthode développée montrent une bonne concordance avec ceux obtenus à l'aide d'une méthode classique, et une meilleure précision du champ de contraintes solution du problème.

ABSTRACT. This paper deals with dual methods for solving unilateral problems with friction. Various variational formulations and their discretizations are presented. The problem is condensed on the contact zone and solved by a Gauss Seidel red-black relaxation algorithm. Numerical results obtained by the dual method are in good agreement with results obtained by classical methods and show better precision on the stress field obtained.

MOTS-CLÉS : contact, frottement, éléments finis «équilibre », formulation duale.

KEYWORDS: contact, friction, equilibrium finite elements, dual formulation. 


\section{Introduction}

Nous présentons des méthodes duales de résolution d'un problème de contact unilatéral avec frottement de Coulomb. Ces méthodes diffèrent des méthodes de résolution traditionnelles car l'inconnue considérée n'est plus le champ des déplacements, mais le champ des contraintes. Ce travail fait suite à (Bisegna et $a l ., 2004)$. Nous présentons ici une démarche systématique permettant la discrétisation des opérateurs, indépendante du type d'élément choisi. Nous proposons par ailleurs, un nouvel algorithme de résolution du système non linéaire obtenu après discrétisation. Cet algorithme a pour avantage sa facilité de mise en œuvre. La méthode duale a été implémentée dans le logiciel LMGC90 (http ://www.lmgc.univmontp2.fr/dubois/LMGC90/). Notre démarche présente l'avantage de pouvoir être mise en place en utilisant bon nombre de modules existants dans les codes d'éléments finis traditionnels. Enfin nous nous intéressons aux résultats obtenus dans le volume, les comparons à ceux obtenus par une méthode primale et montrons le gain de précision obtenu sur la détermination des contraintes.

La section 2 présente le problème de contact unilatéral avec frottement de Coulomb d'un corps élastique sur un obstacle rigide. Différentes formulations variationnelles, ainsi que leurs cadres mathématiques associés sont présentés. La section 3 décrit la discrétisation du problème par éléments finis ; le champ de contraintes devant être statiquement admissible, nous utilisons des éléments finis de type «équilibre », qui permettent de construire le champ de contraintes élémentaire à partir d'une fonction de Airy. Les degrés de liberté élémentaires sont alors les valeurs nodales de la fonction de Airy. Nous présentons comment sont reliés les efforts appliqués et les efforts de contact aux degrés de liberté. La section 4 présente la condensation du problème sur le bord de contact, ainsi que sa résolution par un algorithme de relaxation de type Gauss Seidel rouge-noir (Hackbusch, 1982). Pour finir, dans la section 5, nous présentons les résultats obtenus à l'aide de la méthode développée et les confrontons aux résultats obtenus à l'aide d'une résolution par éléments finis en déplacements. Un résultat de convergence de la méthode des éléments finis «équilibre » est présenté en annexe dans un cadre simplifié.

\section{Position du problème et formulations variationnelles}

\subsection{Présentation du problème}

Dans cette section, nous présentons le problème mécanique et le cadre variationnel. On donne deux formulations variationnelles du problème, l'une écrite dans le volume, l'autre sur la surface. On considère un solide élastique qui occupe l'ouvert $\Omega \subset \mathbb{R}^{d},(d=2,3)$, de frontière suffisamment régulière. La frontière est constituée de trois parties ouvertes disjointes $\Gamma_{D}, \Gamma_{F}$ et $\Gamma_{C}$ telles que $\partial \Omega=\bar{\Gamma}_{D} \cup \bar{\Gamma}_{F} \cap \bar{\Gamma}_{C}$ et mes $\left(\Gamma_{D}\right)>0$. On note $u=\left(u_{i}\right)$ le champ des déplacements, $\epsilon=\epsilon_{i j}(u)=$ $\frac{1}{2}\left(u_{i, j}+u_{j, i}\right)$ le tenseur des déformations linéarisé et $\sigma=\sigma_{i j}(u)=A_{i j k l} \epsilon_{k l}(u)$ le 
tenseur des contraintes. $A$ est borné et vérifie les conditions de symétrie et de positivité usuelles. On note $\alpha=A^{-1}$ le tenseur de souplesse. Le corps est en contact affleurant avec un obstacle rigide sur la partie de frontière $\Gamma_{C}$. Le contact s'effectue avec frottement et est modélisé par la loi de contact unilatéral de Signorini et la loi de pseudofrottement (éventuellement non locale) de Coulomb . Le caractère non local de la loi de Coulomb provient de la régularisation éventuelle de la contrainte normale de contact. Cette régularisation permet d'assurer l'existence et l'unicité de la solution pour un coefficient de frottement suffisamment petit (Cocu, 1984). On emploie le terme pseudofrottement car ici la loi est écrite en déplacement et non pas en vitesse ; on se trouve donc soit dans le cadre d'un chargement monotone, soit à l'intérieur d'un pas de charge pour un chargement plus général. Sur la frontière de contact, le déplacement et le vecteur contrainte sont décomposés en introduisant le vecteur normal $n$ extérieur à $\Gamma$ :

$$
\begin{gathered}
u_{n}=u_{i} n_{i}, u_{t}=u-u_{n} n \\
\sigma_{n}=\sigma_{i j} n_{i} n_{j},\left(\sigma_{t}\right)_{i}=\sigma_{i j} n_{j}-\sigma_{n} n_{i}
\end{gathered}
$$

Le corps est soumis à une densité de forces de volume $F=\left(F_{i}\right)$ dans $\Omega$ et à une densité de forces de surface $f=\left(f_{i}\right)$ sur $\Gamma_{F}$. On suppose que $F \in\left[L^{2}(\Omega)\right]^{d}$ et $f \in\left[L^{2}\left(\Gamma_{F}\right)\right]^{d}$. Sur $\Gamma_{D}$ le déplacement est donné. De façon classique, le problème mécanique d'un corps élastique en contact frottant avec un obstacle rigide, peut s'écrire comme suit :

Problème $(\mathbf{P})$. Trouver le champ des déplacements $u$ et le champ des contraintes $\sigma$ tels que

$$
\left\{\begin{array}{l}
\sigma=A \epsilon(u) \quad \text { dans } \Omega \\
\operatorname{div} \sigma=-F \quad \text { dans } \Omega \\
u=u_{0} \quad \operatorname{sur} \Gamma_{D} \\
\sigma n=f \quad \operatorname{sur} \Gamma_{F} \\
u_{n} \leq 0 \quad, \quad \sigma_{n} \leq 0 \quad, \quad u_{n} \sigma_{n}=0 \quad \operatorname{sur} \Gamma_{C} \\
\left|\sigma_{t}\right| \leq-\mu \sigma_{n}^{*} \operatorname{sur} \Gamma_{C} \text { et }\left\{\begin{array}{l}
\left|\sigma_{t}\right|<-\mu \sigma_{n}^{*} \Rightarrow u_{t}=0 \\
\left|\sigma_{t}\right|=-\mu \sigma_{n}^{*} \Rightarrow \exists \lambda \geq 0, u_{t}=-\lambda \sigma_{t}
\end{array}\right.
\end{array}\right.
$$

où $\mu$ désigne le coefficient de frottement (borné et positif) et $*$ l'opérateur de régularisation normal.

REMARQUE. - L'opérateur * est un opérateur linéaire continu de $H^{-\frac{1}{2}}\left(\Gamma_{C}\right)$ dans $L^{2}\left(\Gamma_{C}\right)$. 
On définit les ensembles des champs cinématiquement et statiquement admissibles :

$$
\begin{aligned}
& V=\left\{v \in\left[H^{1}(\Omega)\right]^{d} ; v=u_{0} \text { p.p. } \operatorname{sur} \Gamma_{D}\right\} \\
& K=\left\{u \in V ; u_{n} \leq 0 \operatorname{sur} \Gamma_{C}\right\} \\
& H=\left\{\sigma=\left(\sigma_{i j}\right), \sigma_{i j}=\sigma_{j i} \in L^{2}(\Omega)\right\} \\
& H_{F, f}=\left\{\sigma \in H ; \sigma_{i j, j}+F_{i}=0 \text { dans } \Omega, \sigma_{i j} n_{j}=f_{i} \operatorname{sur} \Gamma_{F}\right\} \\
& \Sigma(\tau)=\left\{\sigma \in H_{F, f} ; \sigma_{n} \leq 0,\left|\sigma_{t}\right| \leq-\mu \tau_{n} \text { sur } \Gamma_{C}\right\} \\
& C(\xi)=\left\{\tau \in\left(H^{-\frac{1}{2}}\left(\Gamma_{C}\right)\right)^{2} ;|\tau| \leq-\mu \xi\right\} \\
& K_{n}=\left\{U \in H^{\frac{1}{2}}\left(\Gamma_{C}\right) ; U=\gamma u / \Gamma_{C} \text { avec } u \in V, U \leq 0\right\} \\
& K_{n}^{*}=\left\{\tau \in H^{-\frac{1}{2}}\left(\Gamma_{C}\right) ;<\tau, U>\leq 0, \forall U \in K_{n}\right\} \\
& \Sigma^{*}(\xi)=\left(-K_{n}^{*}\right) \times C(\xi)
\end{aligned}
$$

\subsection{Formulation en contraintes ou duale}

La formulation variationnelle en termes de contraintes du problème $(P)$ a été introduite par (Telega, 1991) et s'écrit :

Problème $\left(\mathbf{P}_{\mathbf{S}}\right)$. Trouver un champ de contraintes $\sigma: \Omega \rightarrow H$ tel que

$$
\left\{\begin{array}{l}
\sigma \in \Sigma(\sigma) \\
b(\sigma, \tau-\sigma) \geq l(\tau-\sigma) \quad \forall \tau \in \Sigma(\sigma)
\end{array}\right.
$$

avec $b(\sigma, \tau)=\int_{\Omega} A^{-1} \sigma \cdot \tau d x$

et $l(\tau)=\int_{\Gamma_{D}} u_{0} \tau \cdot n d s$

REMARQUE. - Contrairement à la formulation en déplacements, l'hypothèse de régularité sur la contrainte normale n'est ici plus indispensable pour donner un sens au problème si le coefficient de frottement est assez régulier. Toutefois, cette hypothèse est nécessaire pour assurer l'existence et l'unicité conditionnelle de la solution (Cocu, 1984; Drabla et al., 1999). 


\subsection{Formulation duale condensée}

On introduit formellement deux opérateurs de relèvement (voir par exemple (Quarteroni et al., 1999)), $s \in H^{-1 / 2}\left(\Gamma_{C}\right) \rightarrow \sigma=R(s) \in H_{F, f}$ et $U \in$ $H^{1 / 2}\left(\Gamma_{D}\right) \rightarrow u=T(U) \in V$. Le problème dual condensé s'écrit :

Problème $\left(\mathbf{P}_{\mathbf{d}}^{*}\right)$. Trouver $s=\left(\sigma_{n}, \sigma_{t}\right) \in \Sigma^{*}\left(\sigma_{n}\right)$ tel que

$$
B(s, t-s) \geq L(t-s), \forall t=\left(\tau_{n}, \tau_{t}\right) \in \Sigma^{*}\left(\sigma_{n}\right)
$$

avec $B(s, t)=\int_{\Gamma_{C}} R^{T} A^{-1} R s t d l$

et $L(t)=\int_{\Gamma_{C}} R^{T} T u_{0} R t d l$.

\subsection{Rappels sur la théorie M-CD-M de la dualité}

Comme nous venons de le voir, la formulation duale condensée peut s'obtenir par condensation de la formulation duale (1) sur figure 1), en utilisant des techniques similaires à la condensation classique utilisée pour le problème primal (2) sur figure 1). Elle peut aussi s'obtenir à partir de la formulation primale (3) sur figure 1) en utilisant la théorie de la dualité au sens de Mosco-Capuzzo Dolcetta-Matzeu (notée M-CD-M) (voir (Mosco, 1976; Capuzzo-Dolcetta et al., 1980)). Les deux problèmes obtenus par (1) et (3) sont formellement équivalents. Une démonstration rigoureuse de cette équivalence est, à notre connaissance, un problème ouvert.

En utilisant la théorie M-CD-M, on peut aussi montrer que la formulation primale s'obtient par dualisation, soit de la formulation en contraintes (4) sur figure 1) soit de la formulation en contraintes condensée (5) sur figure 1). Le problème dual condensé peut donc être obtenu par (1) ou (3); toutefois, l'utilisation de la théorie M-CD-M au niveau discret semble plus lourde à mettre en œuvre que la condensation du problème dual (nécessite l'inversion d'une matrice provenant de la discrétisation primale). Par ailleurs, l'obtention d'une solution statiquement admissible (issue de la formulation duale) permet d'obtenir une meilleure précision sur le champ des contraintes.

\section{Discrétisation des formulations en contraintes}

\subsection{Introduction}

Comme nous le verrons par la suite, afin d'obtenir le problème condensé discret, on procède comme suit :

- on discrétise le problème à partir de l'énergie complémentaire; les efforts de contact et de frottement sont introduits dans le problème comme des efforts imposés ;

- la matrice de souplesse et le second membre sont ensuite condensés sur les nœuds de contact ;

- les conditions de contact et de frottement sont imposées sur le problème condensé lors de sa résolution. 


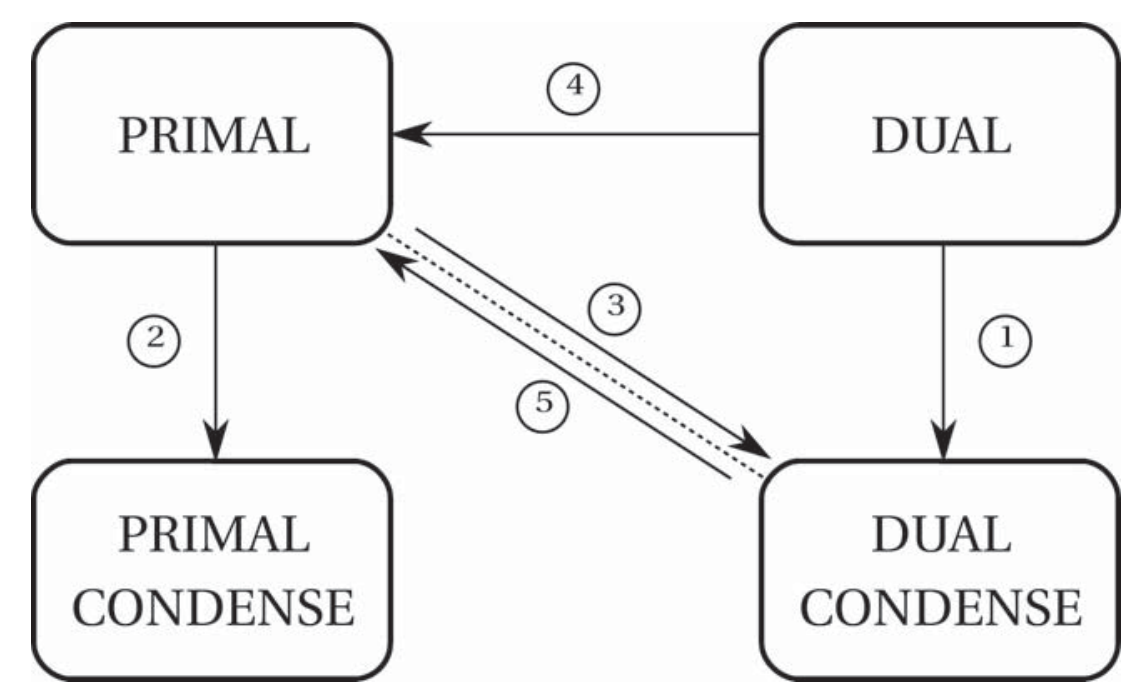

Figure 1. Schéma d'équivalence des formulations

\subsection{Formulation discrète}

Nous présentons dans cette section, la discrétisation du problème formulé en contraintes, à partir de l'écriture du problème sous la forme de l'énergie complémentaire. Le problème est discrétisé par la méthode des éléments finis «équilibre », introduite par (Fraeijs de Veubeke, 2001), et récemment utilisée par (Kempeneers et al., 2003). Le champ de contraintes élémentaire doit satisfaire les conditions d'équilibre local; dans ce but, nous utilisons un élément pour lequel le champ de contraintes dérive d'un potentiel de Airy. Ce type d'élément a été utilisé dans différents cas de calcul numérique : par (Zavelani-Rossi, 2001) dans le cas de structures planes en présence de plasticité et de fissures, par (Wieckowski et al., 1999) pour l'analyse élastoplastique de structures planes, par (Bisegna et al., 2004) pour résoudre le problème de Signorini et Coulomb pour une structure plane élastique. La fonctionnelle de l'énergie complémentaire $\pi$ s'écrit :

$$
\pi=\frac{1}{2} b(\tau, \tau)-l(\tau) .
$$

La discrétisation du problème amène à transformer la fonctionnelle $\pi$ en une somme de fonctionnelles élémentaires. On a alors :

$$
\begin{array}{ll}
\pi=\sum_{e=1}^{N} \pi_{e} \text { avec }: & \pi_{e}=\frac{1}{2} b\left(\tau^{e}, \tau^{e}\right)-l\left(\tau^{e}\right) \text { et } \\
& b\left(\sigma^{e}, \tau^{e}\right)=\int_{\Omega_{e}} \alpha_{i j k l}^{e} \sigma_{i j}^{e} \tau_{k l}^{e} d x_{e} \\
l\left(\tau^{e}\right)=\int_{\Gamma_{D_{e}}} \tau_{i j}^{e} n_{j} u_{0 i} d s_{e} \text { si le bord de l'élément } \in \Gamma_{D}
\end{array}
$$


Sur chaque élément $K$, il faut vérifier la condition d'équilibre local, vérifier la continuité du vecteur contrainte sur les bords des éléments voisins $K^{\prime}$, et imposer les conditions aux limites de type effort si le bord de l'élément appartient à $\Gamma_{F}$ ou $\Gamma_{C}$; soit respectivement :

$$
\begin{aligned}
& d i v \tau^{e}+F=0 \text { sur } K \\
& \tau^{e} n=\tau^{e^{\prime}} n \text { si } \Gamma_{K}=\Gamma_{K^{\prime}} \\
& \tau^{e} n=f \text { si } \Gamma_{K} \in \Gamma_{F} \\
& \tau^{e} n=f_{C} \text { si } \Gamma_{K} \in \Gamma_{C}
\end{aligned}
$$

\subsection{Champ de contraintes élémentaire}

De manière à vérifier la condition [8] a priori, on exprime le champ de contraintes sur $K$ comme dérivant d'une fonction de Airy $\left(\Phi^{e}\right)$, tel que, dans un repère cartésien orthonormé $\left(\Phi^{e}=\Phi^{e}(x, y)\right)$ :

$$
\left\{\begin{array}{l}
\tau_{x x}^{e}=\Phi^{e}(x, y)_{, y y}+\psi \\
\tau_{y y}^{e}=\Phi^{e}(x, y)_{, x x}+\psi \\
\tau_{x y}^{e}=-\Phi^{e}(x, y)_{, x y}
\end{array}\right.
$$

Avec $\psi$ tel que $F i=-\psi_{, i}$, où $F i$ représente les forces de volume dans la direction i (par la suite, le problème sera écrit à forces volumiques nulles de manière à simplifier l'écriture).

Nous utilisons ici l'élément de Bogner-Fox-Schmidt (BFS) présenté figure 2, sur cet élément, la fonction de Airy est interpolée par un polynôme de degrés 3 (voir (Wieckowski et al., 1999)) :

$$
\Phi^{e}(x, y)=\sum_{p, q \leq 3} a_{p q}^{e} x^{p} y^{q}
$$

Nous définissons ce polynôme comme le produit de fonctions de forme $Y^{e}(x, y)$ et des degrés de liberté $\phi^{e}$.

$$
\Phi^{e}=\left[Y^{e}(x, y)\right]\left[\phi^{e}\right]
$$

Les degrés de liberté choisis sont les valeurs nodales de la fonction de Airy et de ses dérivées premières, aux nœuds $P_{i}:\left[\Phi^{e}\left(P_{i}\right) ; \Phi^{e},{ }_{x}\left(P_{i}\right) ; \Phi^{e},{ }_{y}\left(P_{i}\right) ; \Phi^{e},{ }_{x y}\left(P_{i}\right)\right]$. Ce choix permet d'obtenir un polynôme de régularité $C^{1}$ sur les bords de l'élément. La 


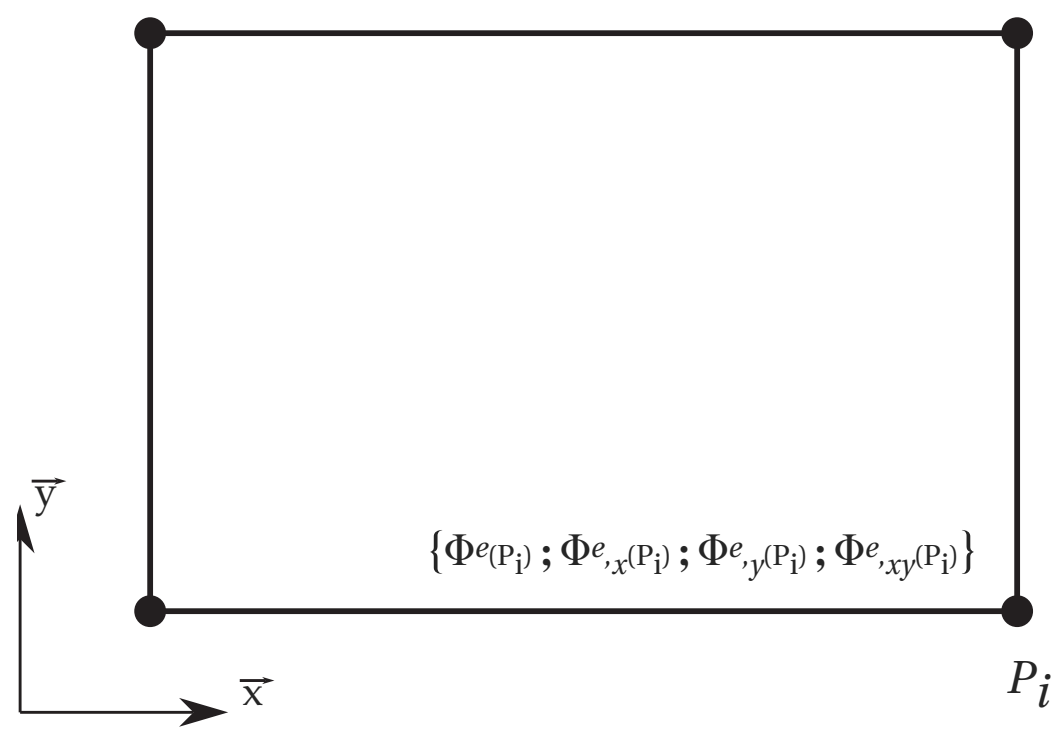

Figure 2. L'élément à 4 næuds et 16 degrés de liberté de Bogner-Fox-Schmit (BFS)

condition [9] de continuité du vecteur contrainte est alors assurée entre éléments. Le champ de contraintes élémentaire est obtenu par dérivation de la fonction de Airy :

$$
\left[\tau^{e}\right]=\left(\begin{array}{c}
\tau_{x x}^{e} \\
\tau_{y y}^{e} \\
\tau_{x y}^{e}
\end{array}\right)=\left[B^{e}\right]\left[\phi^{e}\right]
$$

Il est à noter que cet élément ne convient qu'aux cas géométriquement simples, mais sa simplicité d'implémentation nous a permis d'ouvrir la voie à l'implémentation d'éléments plus complexes dans notre code de calcul. Nous donnons en annexe quelques résultats de convergence de la méthode des éléments finis «équilibre »dans un cadre simplifié.

\subsection{Ecriture du problème élémentaire}

Il reste à imposer les conditions [10] et [11], pour ce faire, on interpole le vecteur contrainte sur un bord de l'élément par :

$$
\tau^{e} n=\left[N^{e}\right]\left[g^{e}\right],
$$

où $\left[N^{e}\right]$ est la matrice des fonctions d'interpolation, dont le degré dépend du type d'élément considéré. Le vecteur $\left[g^{e}\right]$ des efforts généralisés représente donc les valeurs de $\tau^{e} n$ en différents points du bord, dont le nombre dépend du degré de la fonction d'approximation des contraintes. Dans le cas de l'élément BFS, la partie normale du vecteur contrainte est linéaire et la partie tangentielle est quadratique; deux valeurs de 
$\left[g^{e}\right]$ représentent donc la partie normale et trois valeurs de $\left[g^{e}\right]$ représentent la partie tangentielle. On relie ensuite les efforts généralisés aux degrés de liberté :

$$
\begin{array}{r}
{\left[g^{e}(r i)\right]=\tau^{e}\left(r_{i}\right) n=[n]\left[B^{e}\left(r_{i}\right)\right]\left[\phi^{e}\right]} \\
\text { Soit : }\left[g^{e}\right]=\left[C^{e}\right]\left[\phi^{e}\right]
\end{array}
$$

Avec [n] la matrice de projection sur la normale au bord de l'élément, telle que :

$$
\begin{aligned}
& n=\mid \begin{array}{l}
l \\
m
\end{array} \text { dans le repère }(\mathrm{x}, \mathrm{y}) \\
& {[n]=\left[\begin{array}{ccc}
l & 0 & m \\
0 & m & l
\end{array}\right]}
\end{aligned}
$$

Les conditions [10] et [11] sont alors imposées en définissant un vecteur $\left[F^{e}\right]$ (respectivement $\left[F_{C}^{e}\right]$ ) représentant les valeurs discrètes des efforts extérieurs $f$ appliqués aux bords de l'élément (respectivement efforts de contact et frottement $f_{C}$ ), et en les reliant aux degrés de liberté élémentaires :

$$
\begin{aligned}
{\left[F^{e}\right] } & =\left[C_{F}^{e}\right]\left[\phi^{e}\right] \\
{\left[F_{C}^{e}\right] } & =\left[C_{C}^{e}\right]\left[\phi^{e}\right]
\end{aligned}
$$

En tenant compte des définitions précédentes, la fonctionnelle, sous sa forme matricielle, devient :

$$
\pi_{e}=\frac{1}{2} \int_{\Omega_{e}}\left[\tau^{e}\right]^{T}\left[\alpha^{e}\right]\left[\tau^{e}\right] d x_{e}-\int_{\Gamma_{D_{e}}}\left(\left[\tau^{e}\right][n]\right)^{T}[u] d s_{e}
$$

On introduit le vecteur des déplacements généralisés $\left[q^{e}\right]$ sur les bords de l'élément considéré tel que :

$$
\int_{\Gamma_{D_{e}}}\left(\left[\tau^{e}\right][n]\right)^{T}[u] d s_{e}=\left[\phi^{e}\right]^{T}\left[C^{e}\right]^{T} \int_{\Gamma_{D_{e}}}\left[N^{e}\right]^{T}[u] d s_{e}=\left[\phi^{e}\right]^{T}\left[C^{e}\right]^{T}\left[q^{e}\right]
$$

La fonctionnelle s'écrit finalement, en introduisant la matrice de souplesse élémentaire $\left[S^{e}\right]$ :

$$
\begin{aligned}
& \pi_{e}=\frac{1}{2}\left[\phi^{e}\right]^{T}\left[S^{e}\right]\left[\phi^{e}\right]-\left[\phi^{e}\right]^{T}\left[C^{e}\right]^{T}\left[q^{e}\right] \\
& {\left[F^{e}\right]=\left[C_{F}^{e}\right]\left[\phi^{e}\right] \text { si } \Gamma_{K} \in \Gamma_{F}} \\
& {\left[F_{C}^{e}\right]=\left[C_{C}^{e}\right]\left[\phi^{e}\right] \text { si } \Gamma_{K} \in \Gamma_{C}}
\end{aligned}
$$




\section{Système global et résolution}

Nous avons précédemment défini le système élémentaire, le système global s'obtient directement par assemblage et se note :

$$
\begin{aligned}
& \pi=\frac{1}{2}[\phi]^{T}[S][\phi]-[\phi]^{T}[C]^{T}[q] \\
& {\left[C_{F}\right][\phi]=[F] \operatorname{sur} \Gamma_{F}} \\
& {\left[C_{C}\right][\phi]=\left[F_{C}\right] \operatorname{sur} \Gamma_{C}}
\end{aligned}
$$

où les matrices $[S],[C],\left[C_{F}\right],\left[C_{C}\right],[F]$ et $\left[F_{C}\right]$ sont obtenues par assemblage des matrices $\left[S^{e}\right],\left[C^{e}\right],\left[C_{F}^{e}\right],\left[C_{C}^{e}\right],\left[F^{e}\right]$ et $\left[F_{C}^{e}\right] .[\phi]$ représente le vecteur des degrés de liberté du système global.

Les deux dernières conditions [29] et [30] sont prises en compte par l'intermédiaire de multiplicateurs de Lagrange. On obtient alors une fonctionnelle de l'énergie complémentaire augmentée :

$$
\pi^{*}=\frac{1}{2}[\phi]^{T}[S][\phi]-[\phi]^{T}[C]^{T}[q]+[\lambda]\left(\left[C_{F}\right][\phi]-[F]\right)+\left[\lambda^{\prime}\right]\left(\left[C_{C}\right][\phi]-\left[F_{C}\right]\right)
$$

L'annulation de la première variation de cette fonctionnelle par rapport aux variables $\phi, \lambda, \lambda^{\prime}$, et $F_{C}$ nous donne :

$$
\left[\begin{array}{cccc}
S & C_{F}^{T} & C_{C}{ }^{T} & 0 \\
C_{F} & 0 & 0 & 0 \\
C_{C} & 0 & 0 & -I \\
0 & 0 & -I & 0
\end{array}\right]\left[\begin{array}{c}
\phi \\
\lambda \\
\lambda^{\prime} \\
F_{C}
\end{array}\right]=\left[\begin{array}{c}
C^{T} q \\
F \\
0 \\
0
\end{array}\right]
$$

Le système ainsi écrit peut alors être condensé sur le bord de contact. Il se réécrit :

$$
\left[\begin{array}{cc}
A & B^{T} \\
B & 0
\end{array}\right]\left[\begin{array}{c}
\phi_{i} \\
F_{C}
\end{array}\right]=\left[\begin{array}{c}
U_{i} \\
0
\end{array}\right]
$$

où :

$$
\begin{array}{ccc}
{[A]=\left[\begin{array}{ccc}
S & C_{F}^{T} & C_{C}^{T} \\
C_{F} & 0 & 0 \\
C_{C} & 0 & 0
\end{array}\right],[B]=\left[\begin{array}{ccc}
0 & 0 & -I
\end{array}\right]} \\
{\left[\phi_{i}\right]=\left[\begin{array}{c}
\phi \\
\lambda \\
\lambda^{\prime}
\end{array}\right],} & {\left[U_{i}\right]=\left[\begin{array}{c}
C^{T} q \\
F \\
0
\end{array}\right]}
\end{array}
$$


$\phi_{i}$ et $U_{i}$ sont les variables symboliques internes au système condensé (l'équivalent discret du problème $P_{d}^{*}$ ), la condensation consiste à éliminer les variables $\phi_{i}$, le système à résoudre est donc de la forme :

$$
[D]\left[F_{C}\right]=[G]
$$

Avec $[D]=[B]\left[A^{-1}\right]\left[B^{T}\right]$ et $[G]=[B]\left[A^{-1}\right]\left[U_{i}\right]$. On résout ce système non linéaire à l'aide d'un algorithme de type Gauss Seidel rouge-noir. Le vecteur $\left[F_{C}\right]$, de taille $n_{F}$, est structuré de manière à séparer les efforts normaux des efforts tangentiels.

$$
\left[F_{C}\right]=\left[\begin{array}{c}
F_{C}(1) \\
\vdots \\
F_{C}\left(n_{N}\right) \\
- \\
F_{C}\left(n_{N}+1\right) \\
\vdots \\
F_{C}\left(n_{F}\right)
\end{array}\right]=\left[\begin{array}{c}
F_{C_{N}} \\
- \\
F_{C_{T}}
\end{array}\right]
$$

On suit alors l'algorithme de résolution figure 3. Nous définissons le vecteur solution donnée par l'algorithme à l'itération $k: F_{C}^{k}$; l'indicateur de convergence $i_{C}$ est alors calculé, à l'itération $k$, par :

$$
i_{C}^{k}=\frac{\left\|F_{C}^{k}-F_{C}^{k-1}\right\|^{2}}{\left\|F_{C}^{k}\right\|^{2}}
$$

Et nous considérons la convergence atteinte pour $i_{C}$ inférieur à $0,5.10^{-5}$, valeur couramment utilisée pour ce type d'algorithme (Raous et al., 1988).

La séparation des parties normales et tangentielles dans le vecteur $\left[F_{C}\right]$ a pour effet de résoudre successivement deux problèmes de structures plus simples : un problème à frottement donné $\left(i \leq n_{N}\right)$, puis un problème à effort normal donné $\left(i>n_{N}\right)$.

Des preuves de convergence d'un algorithme similaire (par blocs) sont données dans (Bisegna et al., 2001).

\section{Résultats numériques}

La méthode décrite dans les sections précédentes a été implémentée dans le code de calcul LMGC90 (http ://www.lmgc.univ-montp2.fr/dubois/LMGC90/) et appliquée à un benchmark classique de contact avec frottement : le lopin présenté figure 4a est comprimé par les efforts $f_{1}$ et $f_{2}$, et en contact avec un obstacle rigide sur sa partie inférieure. Le problème est traité par symétrie (figure $4 \mathrm{~b}$ ), seuls les résultats sur la partie droite du lopin seront donc présentés. Les caractéristiques du lopin sont : $\mathrm{E}=$ $130 \mathrm{GPa}, \nu=0,2$, sa largeur est de $80 \mathrm{~mm}$, sa hauteur de $40 \mathrm{~mm}$, et l'on fait varier le coefficient de frottement $\mu$ entre 0 et 1 . 
Jusqu'à convergence :

Pour $i=1$ à $n_{F}$ :

Fin de boucle

$$
F_{C}^{*}=\frac{1}{D(i, i)}\left[G(i)-\sum_{j=1}^{i-1} D(i, j) F_{C}(j)-\sum_{j=i+1}^{n_{F}} D(i, j) F_{C}(j)\right]
$$

- Pour $i \leq n_{N}: \mathbf{s i} F_{C}^{*} \geq 0$

- Alors : $F_{C}(i)=0$

- Sinon : $F_{C}(i)=F_{C}^{*}$

- Pour $i>n_{N}: \mathbf{s i}\left|F_{C}^{*}\right|>\mu\left|F_{C_{N}}\right|$

(avec $F_{C_{N}}$ l'effort normal au même point)

- Alors : $F_{C}(i)=\mu$ signe $\left(F_{C}^{*}\right)\left|F_{C_{N}}\right|$

- Sinon : $F_{C}(i)=F_{C}^{*}$

Convergence si $i_{C}^{k}$ petit

\section{Fin de boucle}

Figure 3. Algorithme de résolution
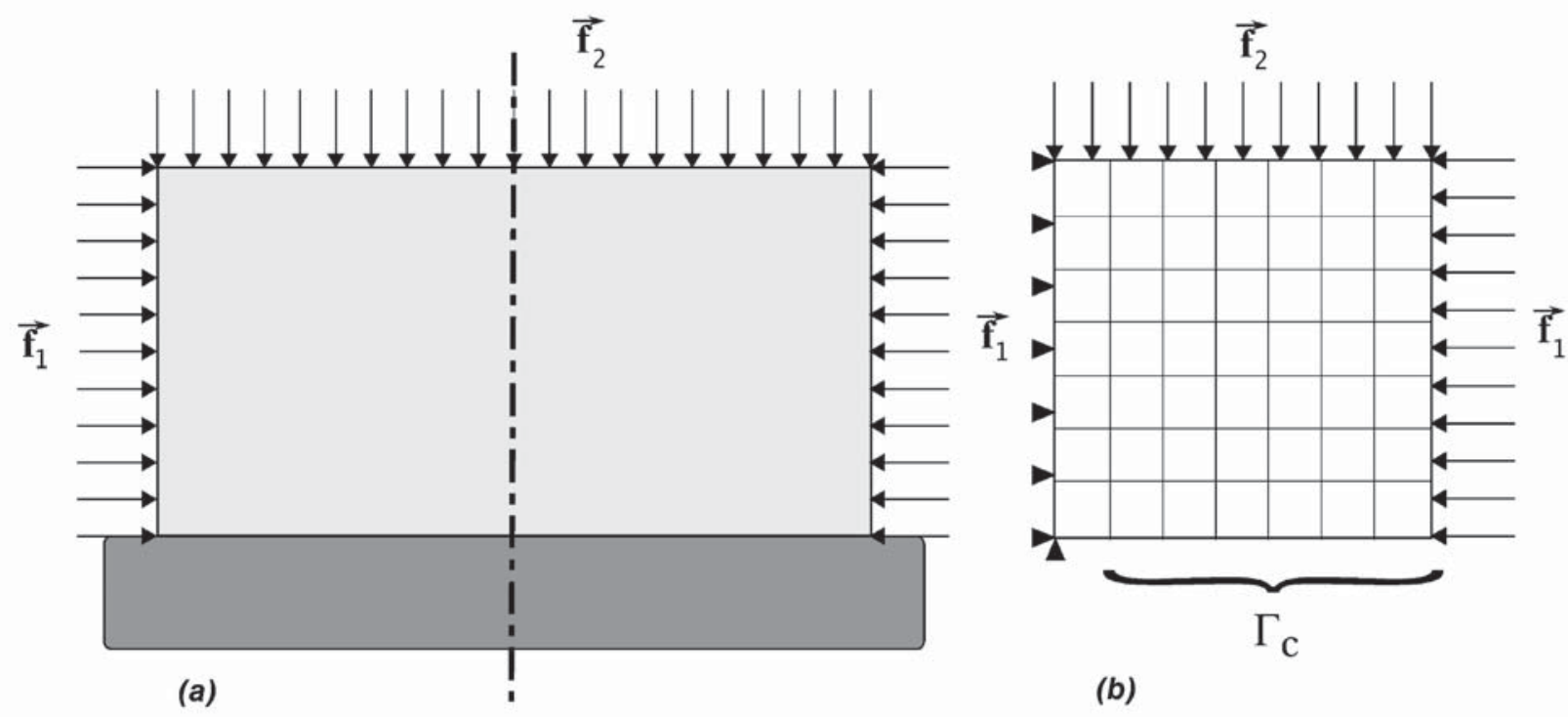

(b)

Figure 4. Le lopin en compression et sa modélisation

\subsection{Comparaison des résultats de contact}

Nous comparons ici les résultats issus de la formulation en contraintes avec ceux issus d'une formulation classique en déplacements (en déformations planes). 
Le maillage du demi-lopin est réalisé, à l'aide de l'élément présenté dans la section 3.3 pour la formulation en contrainte (duale), et par des éléments de type Q4 pour la formulation en déplacements (primale); le maillage adopté présente 32 x 32 éléments dans les deux cas. La figure 5 montre l'évolution des efforts normaux et tangentiels le long de la zone de contact. Les résultats présentés correspondent au cas $f_{1}=15 \mathrm{Mpa}$ $f_{2}=5 \mathrm{Mpa}$ et $\mu=1$, et montrent la bonne concordance des deux méthodes, qui est aussi vérifiée pour d'autres valeurs de $f_{1}, f_{2}$, et $\mu$.

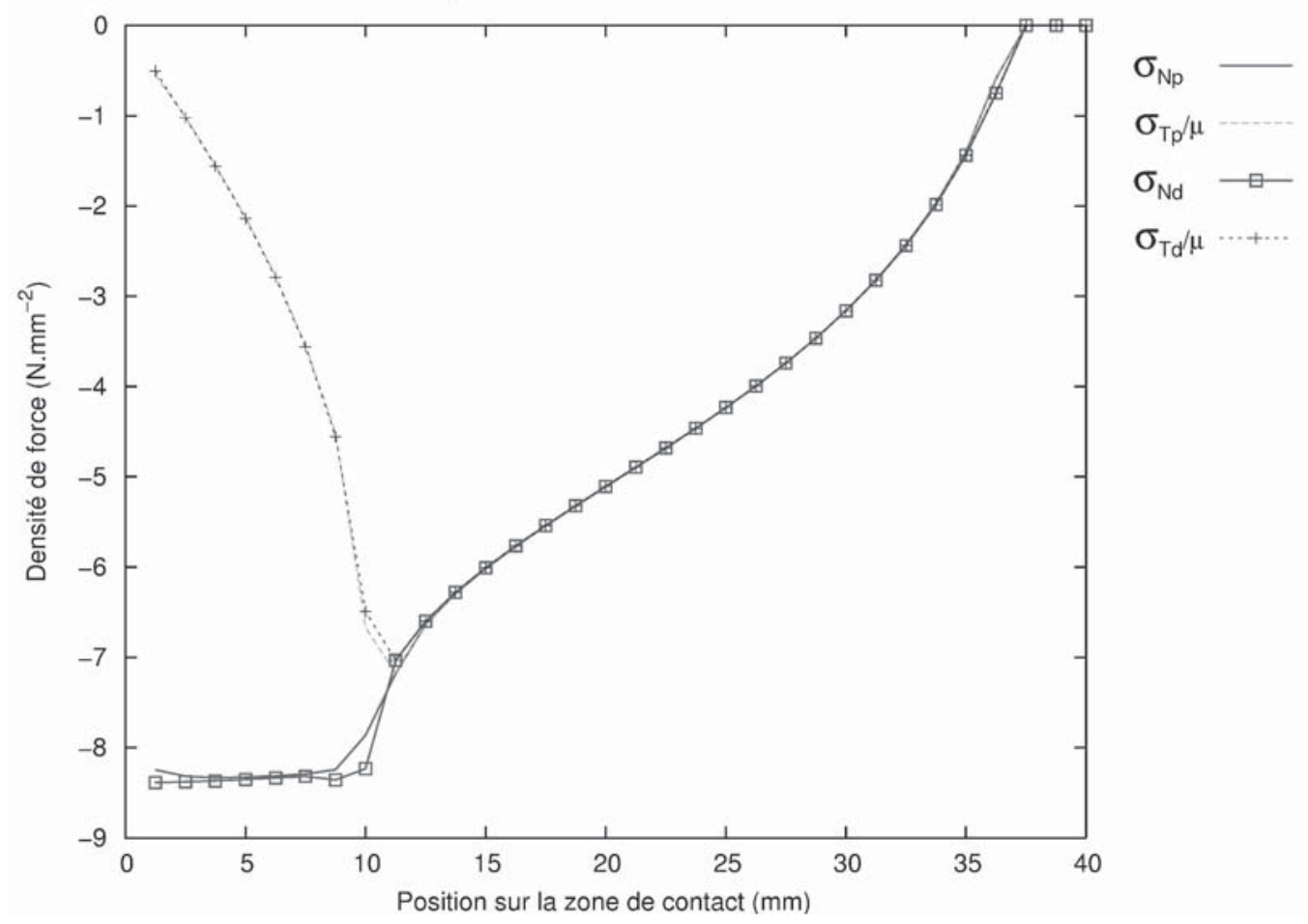

Figure 5. Efforts sur la zone de contact - formulations primale et duale

\subsection{Comparaison des cartes de contraintes}

Dans cette section, nous comparons les champs de contraintes au sein du lopin, obtenus par les approches primale et duale. Les écarts les plus importants ont été remarqués sur la composante $\sigma_{x y}$ du champ de contraintes. De manière à vérifier la convergence des deux types d'approche, nous avons discrétisé la structure avec différentes tailles de maillage. La figure 6 présente les cartes d'isocontraintes $\sigma_{x y}$ pour différentes tailles de maillage. On remarque que les solutions primale et duale convergent vers une même solution, et que les résultats obtenus pour des maillages grossiers sont meilleurs pour l'approche duale que pour l'approche primale.

De manière à quantifier ces écarts, la figure 7 présente, pour les deux types d'approche, le pourcentage d'erreur obtenu sur la contrainte $\sigma_{x y}$. Afin de comparer les approches duale et primale à coût équivalent, les discrétisations sont choisies de manière à avoir des nombres de degrés de liberté proches. Soit un maillage de $3 \times 3$ éléments en dual et $5 \times 5$ en primal; la comparaison s'effectue par rapport à une 
solution de référence correspondant à la solution duale discrétisée par 32 x 32 éléments. Cette analyse montre que les erreurs de l'approche duale sont très inférieures à celles de l'approche primale. Ces résultats s'expliquent d'une part grâce à l'utilisation d'un champ de contraintes statiquement admissible dans le cas dual, de l'autre car les conditions de symétries ne sont pas les mêmes dans les deux cas. En effet, les conditions de symétrie impliquent une contrainte de cisaillement nulle sur le bord de symétrie, et nulle sur les bords où sont appliqués les efforts $f_{1}$ et $f_{2}$; ces conditions sont directement imposées dans le cas dual, alors que les conditions sont imposées sur les déplacements dans le cas primal.
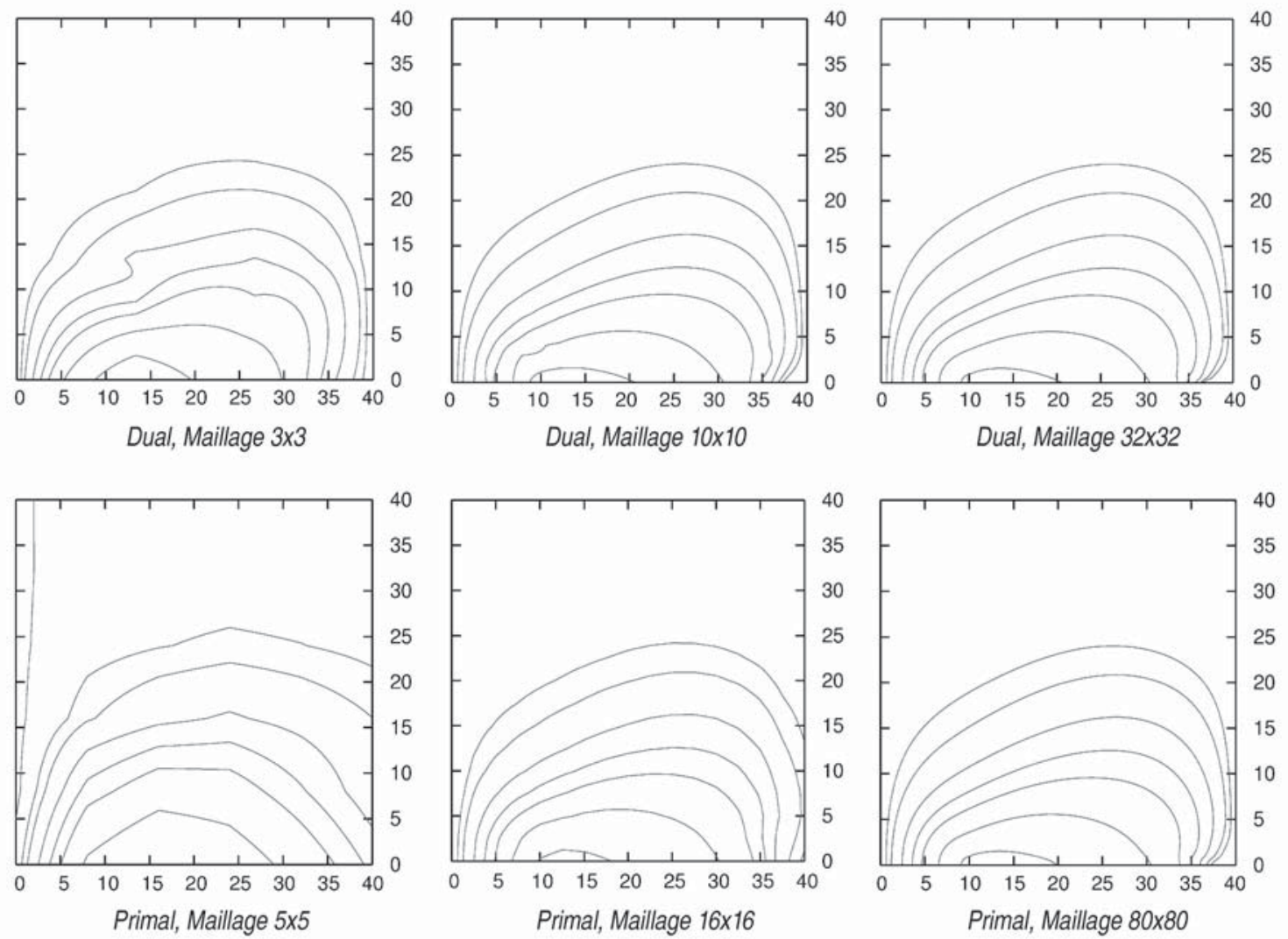

Figure 6. Comparaison de la contrainte $\sigma_{x y}$ pour différents pas de maillage. (Les isocontraintes correspondent, de haut en bas, aux valeurs $0,0,25,0,5,1,1,5,2,3,5$ et 8 Mpa)

\subsection{Convergence de l'algorithme}

Nous analysons dans cette section les facteurs influant sur la rapidité de convergence de l'algorithme proposé. La figure 9 présente l'évolution du nombre d'itérations effectuées jusqu'à convergence en fonction du coefficient de frottement. Quatre cas sont présentés : l'exemple présenté plus haut maillé par 16 x 16 éléments (figure 8a), puis par 5 x 5 éléments (figure 8b) et enfin, le même problème maillé par 16 x 16 éléments avec une géométrie modifiée : le lopin est étiré horizontalement (figure 8c) puis verticalement (figure 8d). Les courbes «Maillage 16 x 16 », « Maillage 5 x 5 », «Maillage 16 x 16 élancé », et «Maillage 16 x 16 élancé* » correspondent respectivement à ces différents cas. 

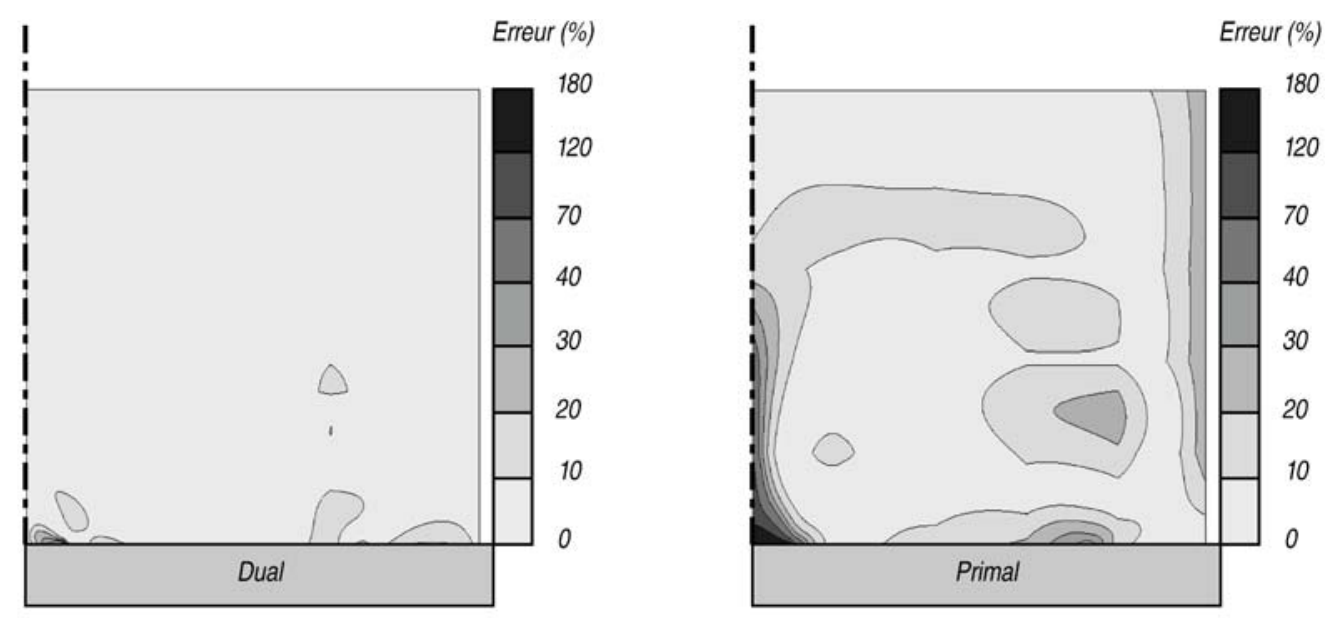

Figure 7. Pourcentage d'erreur sur la contrainte de cisaillement

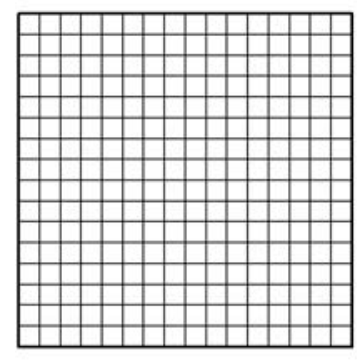

(a)

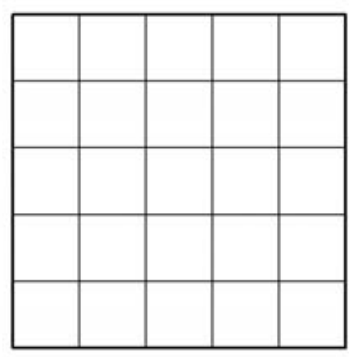

(b)

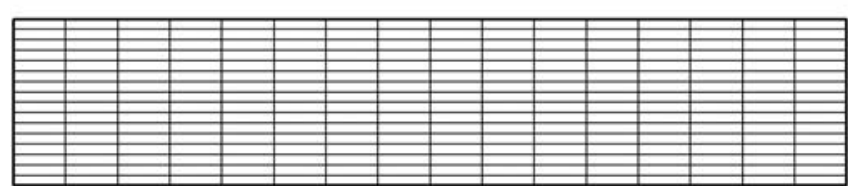

(c)

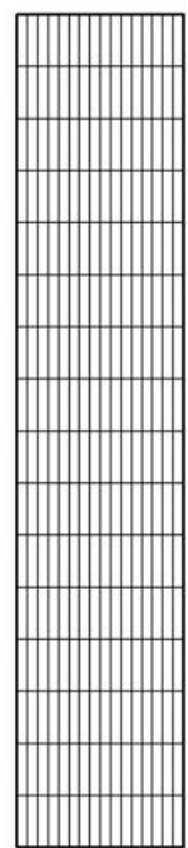

(d)

Figure 8. Quatre maillages : Maillage $16 \times 16(a)$, Maillage $5 \times 5(b)$, Maillage $16 \times$ 16 élancé (c), et Maillage $16 \times 16$ élancé* (d)

On remarque, dans les cas traités, que le coefficient de frottement (dans la gamme considérée) a une faible influence sur la convergence de l'algorithme. Toutefois, dans ces mêmes cas, on peut remarquer que tant que la taille de la surface de contact reste moyenne par rapport aux dimensions de la structure, le frottement a tendance à accélérer la convergence, et qu'une surface de contact importante a tendance à inverser ce phénomène. 


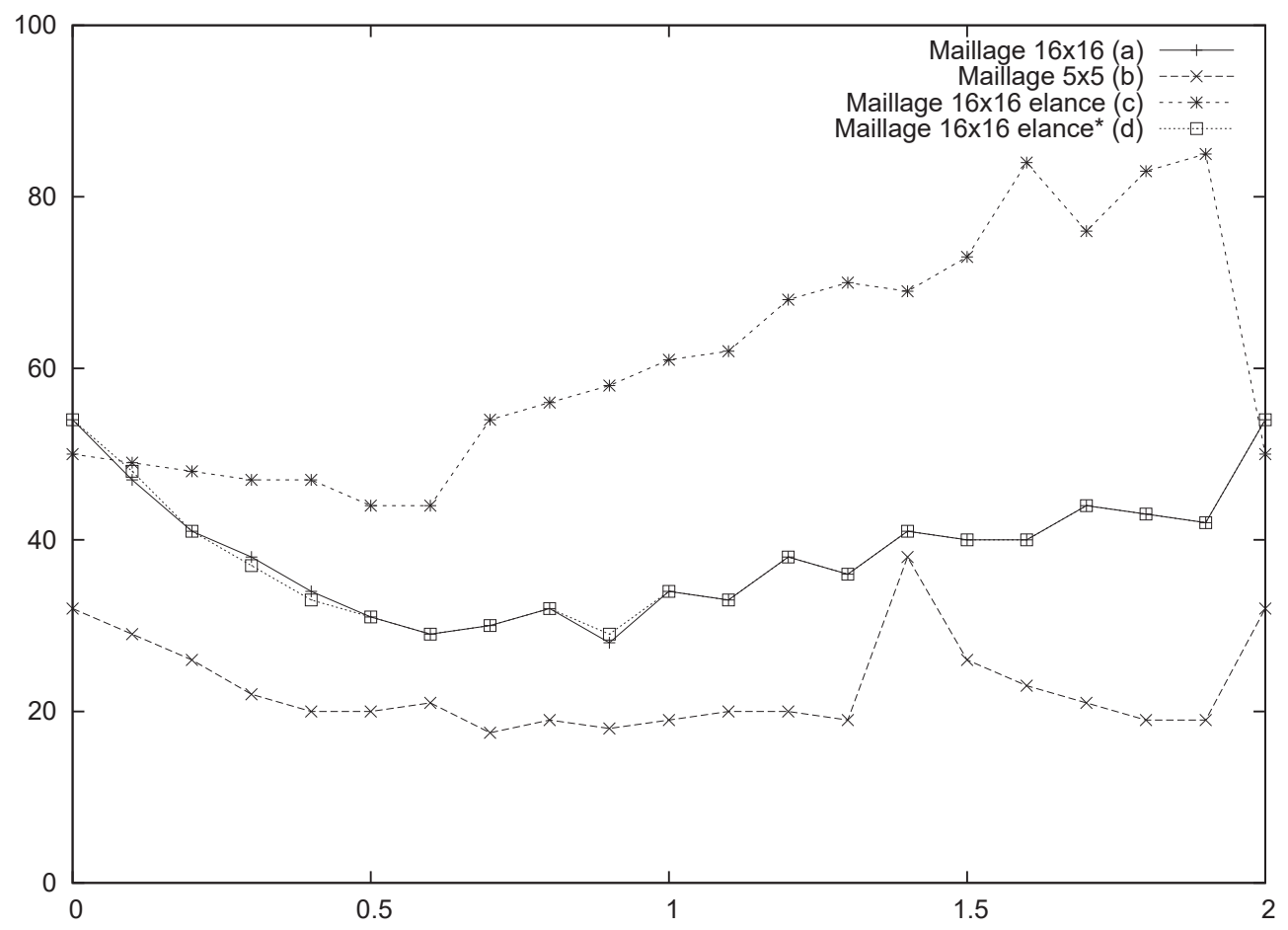

Figure 9. Convergence de l'algorithme - influence du frottement

\section{Conclusion}

Dans cet article, des méthodes duales de résolution d'un problème de contact avec frottement de Coulomb ont été présentées, la discrétisation par éléments finis de type «équilibre »a été adaptée au problème puis condensée et le problème a été résolu par un algorithme de relaxation de type Gauss Seidel rouge-noir. Cette méthode a été implémentée dans le code de calcul LMGC90 et testée sur des exemples académiques.

Les résultats obtenus ont montré la convergence des méthodes duale et primale vers une même solution. La comparaison de ces deux approches a permis de mettre en évidence l'excellente précision des résultats en contraintes obtenus par l'approche duale. Par ailleurs, nous avons constaté la faible dépendance de l'algorithme de résolution aux variations du coefficient de frottement.

Enfin, la simplicité de l'élément présenté nous a permis de développer et de valider notre méthode rapidement mais limite, de par sa géométrie, l'application de la méthode à des cas académiques. Nos études futures viseront donc à développer d'autres types d'éléments et à appliquer la méthode duale aux démarches de raffinement de maillage et d'analyse limite. 


\section{Bibliographie}

Bisegna P., Lebon F., Maceri F., « D-PANA : a convergent block-relaxation solution method for the discretized dual formulation of the Signorini-Coulomb contact problem », ComptesRendus Mathématiques Académie des Sciences Paris, vol. 333, p. 1053-1058, 2001.

Bisegna P., Lebon F., Maceri F., « Relaxation procedures for solving Signorini-Coulomb contact problems », Advances in Engineering Software, vol. 35, p. 595-600, 2004.

Capatina A., Lebon F., " Remarks on the equilibrium finite element method for frictional contact problems », New Trends in Continuum Mechanics, M. Mihailescu-Suliciu, Theta, p. 25-33, 2005.

Capuzzo-Dolcetta I., Matzeu M., « Duality for implicit variational problems and numerical applications », Numerical Functional Analysis and Optimization, vol. 2, p. 231-265, 1980.

Ciarlet P. G., The finite element method for elliptic problems, North Holland publishing company, 1979.

Cocu M., « Existence of solutions of Signorini problems with friction », International Journal of Engineering Science, vol. 22, p. 567-575, 1984.

Drabla S., Sofonea M., « Analysis of a Signorini Problem with Friction », Journal of Applied Mathematics, vol. 62, p. 1-18, 1999.

Fraeijs de Veubeke B., « Displacement and equilibrium Models in the Finite Element Method», International Journal for Numerical Methods in Engineering, vol. 52, p. 287-342, 2001.

Hackbusch W., « Multigrid method of the second kind », Springer, Berlin, 1982.

Kempeneers M., Beckers P., de Almeida J. M., Pereira O. J. A., « Modèles équilibre pour l'analyse duale», Revue européenne des éléments finis, vol. 12, p. 737-760, 2003.

Mosco U., Implicit variational problems and quasivariational inequalities, Lecture Notes in Mathematics, Springer Verlag, 1976.

Quarteroni A., Valli A., Domain decomposition methods for partial differential equations, University Press, Oxford, 1999.

Raous M., Chabrand P., Lebon F., « Numerical methods for solving unilateral contact problem with friction », numéro spécial Journal de Mécanique Théorique et Appliquée, vol. 7 , p. 111-128, 1988.

Telega J., « Quasi-static Signorini's contact problem with friction and duality », International Series on Numerical Mathematics, vol. 101, p. 199-214, 1991.

Wieckowski Z., Youn S.-K., Moon B.-S., « Stress based finite element analysis of plane plasticity problems », International Journal for Numerical Methods in Engineering, vol. 44, p. $1505-1525,1999$.

Zavelani-Rossi A., « An equilibrium approach to plane problems », Computers and Structures, vol. 79, p. 1877-1895, 2001. 


\section{Annexe}

\section{Quelques résultats de convergence de l'interpolation}

Dans cette section, on donne quelques résultats de convergence de la méthode des éléments finis dans un cadre simplifié. On introduit l'espace

$$
W=H(\operatorname{div} ; \Omega)=\left\{\tau \in H ; \operatorname{div} \tau \in\left[L^{2}(\Omega)\right]^{2}\right\} .
$$

Pour simplifier le problème, on suppose que le domaine $\Omega$ est polygonal. On note $\mathrm{T}_{h}$ une triangulation de $\Omega$ telle que $\Omega=\bigcup_{K \in \mathrm{T}_{h}} K$. On suppose que $\mathrm{T}_{h}$ est régulier, c'est-à-dire que tous les angles des éléments $K \in \mathrm{T}_{h}$ sont bornés inférieurement par zéro et qu'il existe une constante positive $\delta$ telle que la longueur des côtés de chaque $K \in \mathrm{T}_{h}$ soit au moins égale à $\delta h$.

Dans un cadre assez général, les espaces $W_{h}$ et $V_{h}$ sont choisis de la façon suivante :

$$
\text { Si } \tau \in W_{h} \text { et }\left(\operatorname{div} \tau, v_{h}\right)=0, \forall v_{h} \in V_{h} \quad \text { alors } \quad \operatorname{div} \tau=0 \text { dans } \Omega
$$

On suppose de plus que l'on est capable de construire un opérateur d'interpolation $\pi_{h}: W \rightarrow W_{h}$ qui satisfait :

$$
\left(\operatorname{div} \pi_{h} \sigma, v_{h}\right)=\left(\operatorname{div} \sigma, v_{h}\right), \quad \forall v_{h} \in V_{h}
$$

Nous allons définir un élément qui vérifie les hypothèses précédentes. Pour tout $K \in \mathrm{T}_{h}$, on définit l'espace de dimension fini :

$$
W_{K} \subset H(d i v ; K)
$$

des tenseurs des contraintes linéaires par morceaux et on pose

$$
W_{h}=\left\{\tau \in \hat{W}_{h} ; \operatorname{div} \tau \in\left[L^{2}(\Omega)\right]^{2}\right\}
$$

avec

$$
\hat{W}_{h}=\left\{\tau \in H ; \tau / K \in W_{K}, \forall K \in \mathrm{T}_{h}\right\} .
$$

Nous ferons un certain nombre d'hypothèses restrictives. On suppose que $F$ est constante (le poids par exemple) et $f$ linéaire (distribution linéaire de forces). Pour le cas du frottement de Tresca, dans les problèmes $\left(P_{s}\right)$ et $\left(P_{d}^{*}\right)$ les ensembles $\Sigma(\sigma)$ et $\Sigma^{*}\left(\sigma_{n}\right)$ sont remplacés respectivement par les ensembles $\Sigma(\beta)$ et $\Sigma^{*}\left(\beta_{n}\right)$ où $\beta$ et $\beta_{n}$ sont donnés. On va supposer de plus, que $\beta$ (resp. $\beta_{n}$ ) est soit concave soit linéaire par morceaux. En utilisant un résultat classique dû à (Ciarlet, 1979), on peut obtenir 
Théorème 1 . Elasticité : il existe une constante $C$ indépendante de h telle que :

$$
\left\|\sigma-\sigma_{h}\right\|_{W} \leq C h\|\sigma\|_{2}
$$

Tresca $\left(P_{s}\right)$ il existe une constante $C$ indépendante de h telle que :

$$
\left\|\sigma-\sigma_{h}\right\|_{W} \leq C h^{1 / 2}\|\sigma\|_{2}
$$

Tresca $\left(P_{d}^{*}\right)$ il existe une constante $C$ indépendante de $h$ telle que :

$$
\left\|\sigma-\sigma_{h}\right\|_{-1 / 2, \Gamma_{C}} \leq C h^{1 / 2}\|\sigma\|_{2}
$$

Les notations \|\|$_{2},\|\|_{W}$ et \|\|$_{-1 / 2, \Gamma_{C}}$ correspondent aux normes usuelles sur les espaces considérés. Ces résultats sont démontrés dans (Capatina et al., 2005).

Remarque. - Pour un problème de Signorini-Coulomb, les hypothèses du théorème 1 ne sont pas vérifiées en général. Il est nécessaire de considérer des approximations externes (Ciarlet, 1979). Ce problème est, à notre connaissance, un problème ouvert. 